International Electronic Journal of Algebra

Volume 31 (2022) 230-242

DOI: $10.24330 /$ ieja.1058435

\title{
ON BELL POLYNOMIALS ASSOCIATED TO VASYUNIN COTANGENT SUMS
}

\author{
Samir Belhadj and Mouloud Goubi \\ Received: 9 May 2021; Revised: 28 August 2021; Accepted: 15 September 2021
}

Communicated by Abdullah Harmanc1

\begin{abstract}
The present work is focused on the study of a cotangent sum associated to the zeros of the Estermann zeta function and Riemann zeta function. We use Bell polynomials and generating functions approach to give arithmetical proof of its Dirichlet series different from that given by M. Th. Rassias.
\end{abstract}

Mathematics Subject Classification (2020): 11F20, 11E45, 11M26

Keywords: Vasyunin cotangent sums, Riemann hypothesis, Bell polynomial

\section{Introduction}

For rational number $p / q$ with $(q, p)=1$ and $q>p>0$, the Vasyunin cotangent sum $V(p / q)[24]$ is defined by

$$
V(p / q)=\sum_{k=1}^{q-1}\left\{\frac{p k}{q}\right\} \cot \frac{\pi k}{q} .
$$

$V(p / q)$ is a periodic function of period 1 and its value is an algebraic number. It is a generalization of Dedekind sums with which it shares the law of reciprocity $[3,13]$. The Vasyunin cotangent sums appear in the study of the Riemann hypothesis through the following identity of the Vasyunin formula [24]:

$$
\begin{aligned}
\int_{0}^{+\infty}\left\{\frac{t}{p}\right\}\left\{\frac{t}{q}\right\} \frac{d t}{t^{2}} & =\frac{\log 2 \pi-\gamma}{2}\left(\frac{1}{p}+\frac{1}{q}\right)+\frac{q-p}{2 p q} \log \frac{p}{q} \\
& -\frac{\pi}{2 p q}(V(p / q)+V(q / p)) .
\end{aligned}
$$

The above formula is related to the Báez-Duarte-Balazard approach [2] to the Riemann hypothesis. The Riemann zeta function is defined by Dirichlet series

$$
\zeta(s)=\sum_{n \geq 1} \frac{1}{n^{s}},
$$

for whom the Euler product formula is

$$
\zeta(s)=\prod_{p: \text { prime }} \frac{1}{1-p^{-s}} .
$$


The zeta function is also found in other areas of algebra such as group and ring theory. We can cite zeta function of a finitely generated group [14,25], Hasse Zeta Functions of Non-commutative Rings [9] and zeta function in integral Representation Theory [23]. All these functions are expressed as product or sum of infinite numbers. Almost they keep the same properties as in analytical number theory. The sum $V(p / q)$ is closely connected with the cotangent sum

$$
c_{0}(p / q)=-\sum_{k=1}^{q-1} \frac{k}{q} \cot \left(\frac{\pi k p}{q}\right)
$$

by virtue of the relation

$$
V(p / q)=-c_{0}(\bar{p} / q)
$$

where $\bar{p}$ is the inverse of $p$ modulo $q$. The sum $c_{0}(p / q)$ appears in the Estermann zeta function $E(s, p / q, \alpha)[15,20]$ defined by the Dirichlet series

$$
E(s, p / q, \alpha)=\sum_{n \geq 1} \frac{\sigma_{\alpha}(n) \exp (2 \pi i n p / q)}{n^{s}},
$$

where $\sigma_{\alpha}(n)=\sum_{d \mid n} d^{\alpha}$. At $s=\alpha=0$ we have

$$
E(0, p / q, 0)=\frac{1}{4}+\frac{i}{2} c_{0}(p / q)
$$

which means that

$$
c_{0}(p / q)=-\frac{1}{2 i}+\frac{2}{i} \sum_{n \geq 1} d(n) \exp (2 \pi i n p / q)
$$

with $d(n)=\sigma_{0}(n)=\sum_{d \mid n} 1$ is the divisor function which counts the number of divisors of $n$. The writing of $c_{0}(p / q)$ as a function of $c_{0}(1 / q)$ is given by the relation (see [17, Proposition 3.2] and [20, Proposition 3.2.2])

$$
c_{0}(p / q)=\frac{1}{p} c_{0}(1 / q)-\frac{1}{p} \sum_{k=1}^{q-1} \cot \left(\frac{\pi k p}{q}\right)\left\lfloor\frac{k p}{q}\right\rfloor .
$$

M. Th. Rassias $[20,21]$ proved the following Dirichlet series of $c_{0}(1 / q)$ :

$$
c_{0}(1 / q)=\frac{1}{\pi} \sum_{\substack{n \geq 1 \\ q \nmid n}}\left[\frac{q}{n}\left(1+2\left\lfloor\frac{n}{q}\right\rfloor\right)-2\right\rfloor .
$$

Separately M. Goubi et al. [13] investigated the sum $V(1 / q)$ and showed that

$$
V(1 / q)=-\frac{1}{\pi} \int_{0}^{1}\left((q-2) t^{q}-q t^{q-1}+q t-q+2\right) f_{q}(t) d t,
$$

where

$$
f_{q}(t)=\frac{1}{(1-t)^{2}\left(1-t^{q}\right)}
$$


It is easily checked that

$$
(q-2) t^{q}-q t^{q-1}+q t-q+2=(t-1)^{3} \sum_{r=1}^{q-1}(q-r-1) r t^{r-1},
$$

and then

$$
V(1 / q)=-\frac{1}{\pi} \int_{0}^{1} \frac{\sum_{r=1}^{q-1}(q-r-1) r t^{r-1}}{1+t+\cdots+t^{q-1}} d t .
$$

$f_{q}$ is a generating function, it is written under the form

$$
f_{q}(t)=\sum_{n \geq 0} b_{q}(n) t^{n},|t|<1,
$$

with $b_{q}(n)$ is the sequence of positive integers defined by the recurrence relation[10]. Using this sequence, we transformed the integral (2) to the following series expansion of $V(1 / q)$ :

$$
V(1 / q)=-\frac{1}{\pi} q(q-1)(q-2) \sum_{n \geq 0} \frac{b_{q}(n)}{(n+1)(n+2)(n+q)(n+q+1)} .
$$

We refer to $[6,7,18]$ for recent algebraic developments of various formal series such as $Z$-transform and Fourier series in symbolic calculus. We remember that $Z$ transform is defined as sum of an infinite number of addends and used for example as a controllable way of solving linear, constant-coefficient difference equations [19]. The idea contained in the $Z$-transform is also not far from the method of generating functions as introduced by de Moivre in probability theory [16]. To explicitly calculate the sequence $b_{q}(n)$ we first define the divisor function $d_{q}(n)$ by the relation

$$
d_{q}(n)=\left\{\begin{array}{cc}
1 & \text { if } q \mid n \\
0 & \text { otherwise }
\end{array}\right.
$$

Let $r$ be the remainder obtained when $n$ is divided into $q$. Moreover, $r=0$ if, and only if, $q \mid n$, which justifies the expression

$$
d_{q}(n)=I(r+1)
$$

where $I$ is the so-called identity arithmetical function [1] which takes the form

$$
I(n)=\left\lfloor\frac{1}{n}\right\rfloor= \begin{cases}1 & \text { if } n=1 \\ 0 & \text { if } n>1\end{cases}
$$

The divisor function $d(n)$ is related to this function by the relation

$$
d(n)=\sum_{k=1}^{n} d_{k}(n)
$$


Therefore we can write $b_{q}(n)$ in the form

$$
b_{q}(n)=\sum_{k=0}^{n}(n-k+1) d_{q}(k) .
$$

The proof consists to write

$$
f_{q}(t)=\left(\sum_{n \geq 0}(n+1) t^{n}\right)\left(\sum_{n \geq 0} d_{q}(n) t^{n}\right)
$$

and the result follows from the Cauchy product of generating functions. A simple calculation shows that

$$
\sum_{k=0}^{n}(n-k+1) d_{q}(k)=\sum_{j=0}^{\lfloor n / q\rfloor}(n-j q+1),
$$

and then

$$
\sum_{k=0}^{n}(n-k+1) d_{q}(k)=(n+1)\left(\left\lfloor\frac{n}{q}\right\rfloor+1\right)-\frac{q}{2}\left\lfloor\frac{n}{q}\right\rfloor\left(\left\lfloor\frac{n}{q}\right\rfloor+1\right) .
$$

Finally we have

$$
b_{q}(n)=\left(n+1-\frac{q}{2}\left\lfloor\frac{n}{q}\right\rfloor\right)\left(\left\lfloor\frac{n}{q}\right\rfloor+1\right) ;
$$

which is a special case of [4, Proposition 4] for $\bar{p}=1$. If $q \mid n$, it is obvious to remark that

$$
2 b_{q}(n)=(n+2)\left(\frac{n}{q}+1\right) \text { and } b_{q}(n) \equiv\left(\frac{n}{q}+1\right) \quad(\bmod p) .
$$

The exponential partial Bell polynomials [5] $B_{n, k}:=B_{n, k}\left(x_{1}, \cdots, x_{n-k+1}\right)$ are defined by the generating function

$$
\frac{1}{k !}\left(\sum_{n \geq 1} x_{n} \frac{t^{n}}{n !}\right)^{k}=\sum_{n \geq k} B_{n, k} \frac{t^{n}}{n !}
$$

and admit for explicit formula the expression

$$
B_{n, k}=\frac{n !}{k !} \sum_{s_{n}(k)}\left(\begin{array}{c}
k \\
k_{1}, \cdots, k_{n-k+1}
\end{array}\right) \prod_{r=1}^{n-k+1}\left(\frac{x_{r}}{r !}\right)^{k_{r}},
$$

where $s_{n}(k)$ is the set of all $k_{1}, k_{2}, \cdots, k_{n-k+1}$ for which $k_{1}+k_{2}+\cdots+k_{n-k+1}=k$ and $k_{1}+2 k_{2}+\cdots+n k_{n-k+1}=n$, and

$$
\left(\begin{array}{c}
k \\
k_{1}, \cdots, k_{n-k+1}
\end{array}\right)=\frac{k !}{k_{1} ! \cdots k_{n-k+1} !} .
$$

The following are some particular values $B_{n, k}[5]$ :

$$
B_{n, k}(1,1, \cdots)=S(n, k),(\text { Stirling numbers of second kinds), }
$$




$$
\begin{gathered}
B_{n, k}(1 !, 2 !, \cdots)=\frac{n !}{k !}\left(\begin{array}{c}
n-1 \\
k-1
\end{array}\right), \text { (Lah numbers), } \\
B_{n, k}(1,2, \cdots)=\left(\begin{array}{l}
n \\
k
\end{array}\right) k^{n-k}, \text { (idempotent numbers). }
\end{gathered}
$$

The Bell polynomials are used to calculate the coefficients of a generating function resulting from the composition of two generating functions. Let $f(t)=\sum_{n \geq 0} a_{n} t^{n}$ and $g(t)=\sum_{n \geq 0} b_{n} t^{n}$ be two generating functions and we consider $x_{j}=j ! b_{j}$. The series expansion of $f \circ g$ is given by the expression [11]:

$$
f \circ g(t)=f\left(b_{0}\right)+\sum_{n \geq 1} \sum_{k=1}^{n} B_{n, k} f^{(k)}\left(b_{0}\right) \frac{t^{n}}{n !} .
$$

Consequently for $b_{0}=0$ we have

$$
f \circ g(t)=a_{0}+\sum_{n \geq 1} \sum_{k=1}^{n} k ! a_{k} B_{n, k} \frac{t^{n}}{n !} .
$$

For the special case $f(t)=t^{\alpha}$, where $\alpha \in \mathbb{C}$ and $b_{0} \neq 0$, the identity (7) is written under the form

$$
g^{\alpha}(t)=b_{0}^{\alpha}+\sum_{n \geq 1} \sum_{k=1}^{n}(\alpha)_{k} b_{0}^{\alpha-k} B_{n, k} \frac{t^{n}}{n !} .
$$

For the proof we refer to [12] and references therein. Otherwise $B_{n, k}$ appear in the Faà di Bruno formula [8] for computing the $n$-th derivative of $f \circ g$. Let $D^{n} f$ the $n$-th derivative of $f$ then

$$
D^{n} f(g(t))=\sum_{k=0}^{n}\left(D^{k} f\right)(g(t)) B_{n, k}\left(D^{1} g(t), D^{2} g(t), \cdots\right) .
$$

We refer to [22] for a detailed proof. In this work we purpose a new reformulation of $b_{q}(n)$ based on Bell polynomials and we revisit the proof of the Dirichlet series of $c_{0}(1 / q)$.

\section{Bell polynomials associated to the inverse of generating functions}

When $b_{0} \neq 0 ; 1 / g(t)$ is a generating function. We can always assume $b_{0}=1$ and we take $x_{k}=k ! b_{k}$. Substitute $\alpha=-1$ in the identity (9) to obtain

$$
1 / g(t)=1+\sum_{n \geq 1} \sum_{k=1}^{n}(-1)^{k} k ! B_{n, k} \frac{t^{n}}{n !} .
$$

Let $a_{n}$ be the sequence

$$
a_{n}=\frac{1}{n !} \sum_{k=1}^{n}(-1)^{k} k ! B_{n, k}
$$


with the first term $a_{0}=1$. Thus

$$
g(t) \sum_{n \geq 0} a_{n} t^{n}=1
$$

By means of Cauchy product of generating function we deduce that

$$
\sum_{u=0}^{n} a_{u} b_{n-u}=0, n \geq 1
$$

and we have already proved the following theorem.

Theorem 2.1. The sequence $b_{n}$ satisfies the inversion relation

$$
\sum_{u=1}^{n} \frac{1}{u !} b_{n-u} \sum_{k=1}^{u}(-1)^{k} k ! B_{u, k}=-b_{n}
$$

The identity (11) is useful to show for example that

$$
\begin{gathered}
\sum_{u=1}^{n} \sum_{k=1}^{u}(-1)^{k}\left(\begin{array}{l}
u-1 \\
k-1
\end{array}\right)=-1, \\
\sum_{u=1}^{n}\left(\begin{array}{l}
n \\
u
\end{array}\right) \sum_{k=1}^{u}(-1)^{k} k ! S(n, k)=-1
\end{gathered}
$$

and

$$
\sum_{u=1}^{n} \sum_{k=1}^{u}(-1)^{k} k !\left(\begin{array}{l}
n \\
k
\end{array}\right) C_{n, k} k^{n-k}=-1
$$

where

$$
C_{n, u}=\left\{\begin{array}{cl}
\left(\begin{array}{c}
n-1 \\
u
\end{array}\right) & \text { if } u<n, \\
\frac{1}{n} & \text { if } u=n .
\end{array}\right.
$$

2.1. New reformulation of the sequence $b_{q}(n)$. First we began by relating restricted divisor function $d(n)$ to $b_{q}(n)$ and deduce new reformulation of $d(n)$ by means of the integer part function $\lfloor$.$\rfloor . According to the expression of f_{q}(t)$ we can write

$$
\left(1-2 t+t^{2}\right) f_{q}(t)=\frac{1}{1-t^{q}} \text { and }\left(1-t^{q}\right) f_{q}(t)=\frac{1}{(1-t)^{2}} .
$$

Let $b_{n}$ the sequence defined by $b_{0}=1, b_{1}=-2, b_{2}=1$ and the others are zeros. By means of the Cauchy product of generating functions we obtain respectively

$$
d_{q}(n)=\sum_{k=0}^{n} b_{k} b_{q}(n-k)
$$

and

$$
f_{q}(t)-t^{q} f_{q}(t)=\sum_{n \geq 0}(n+1) t^{n}
$$


Furthermore for $n \geq 2$ we have

$$
d_{q}(n)=b_{q}(n)-2 b_{q}(n-1)+b_{q}(n-2)
$$

and

$$
n+1=b_{q}(n)-b_{q}(n-q), n \geq q
$$

The reformulation of the identities (12) and (13) by means of the integer part function gives

$$
\begin{aligned}
d_{q}(n) & =\left(n+1-\frac{q}{2}\left\lfloor\frac{n}{q}\right\rfloor\right)\left(\left\lfloor\frac{n}{q}\right\rfloor+1\right) \\
& -2\left(n-\frac{q}{2}\left\lfloor\frac{n-1}{q}\right\rfloor\right)\left(\left\lfloor\frac{n-1}{q}\right\rfloor+1\right) \\
& +\left(n-1-\frac{q}{2}\left\lfloor\frac{n-2}{q}\right\rfloor\right)\left(\left\lfloor\frac{n-2}{q}\right\rfloor+1\right)
\end{aligned}
$$

and

$n+1=\left(n+1-\frac{q}{2}\left\lfloor\frac{n}{q}\right\rfloor\right)\left(\left\lfloor\frac{n}{q}\right\rfloor+1\right)-\left(n-q+1-\frac{q}{2}\left\lfloor\frac{n-q}{q}\right\rfloor\right)\left(\left\lfloor\frac{n-q}{q}\right\rfloor+1\right)$.

From another point of view, the expression of $f_{q}(t)$ is written under the form $f_{q}=1 / A_{q}(t)$ where

$$
A_{q}(t)=1-2 t+t^{2}-t^{q}+2 t^{q+1}-t^{q+2} .
$$

One can extend the sum at infinity and obtain $A_{q}(t)=\sum_{n \geq 0} v_{n} t^{n}$, with $v_{n}$ is the sequence defined by $v_{0}=1, v_{1}=-2, v_{2}=1, v_{q}=-1, v_{q+1}=2, v_{q+2}=-1$ and the others are zeros. Let $s_{q}$ be the set $s_{q}=\{1,2, q, q+1, q+2\}$ and $s_{q}(k, n)$ the set of all $\left(k_{i}\right)_{i \in s_{q}}$ for which

$$
k_{1}+k_{2}+k_{q}+k_{p+1}+k_{q+2}=k
$$

and

$$
k_{1}+2 k_{2}+q k_{q}+(q+1) k_{q+1}+(q+2) k_{q+2}=n .
$$

The explicit formula of $b_{q}(n)$ by means of Bell polynomials is given by the following theorem

Theorem 2.2. Letting $x_{j}=j ! v_{j}$, then we have $b_{q}(0)=1$ and for $n \geq 1$;

$$
b_{q}(n)=\sum_{k=1}^{n} \frac{1}{n !}(-1)^{k} k ! B_{n, k} .
$$

Explicitly we have

$$
b_{q}(n)=\sum_{k=1}^{n} \sum_{s_{q}(k, n)}(-1)^{k_{2}+k_{q+1}} 2^{k_{1}+k_{q+1}}\left(\begin{array}{c}
k \\
k_{i}
\end{array}\right)_{i \in s_{q}},
$$


where

$$
\left(\begin{array}{c}
k \\
k_{i}
\end{array}\right)_{i \in s_{q}}=\frac{k !}{k_{1} ! k_{2} ! k_{q} ! k_{q+1} ! k_{q+2} !} .
$$

Proof. It is obvious that

$$
1 / A_{q}(t)=1+\sum_{n \geq 1} \sum_{k=1}^{n}(-1)^{k} k ! B_{n, k} \frac{t^{n}}{n !} .
$$

To get explicit formula of $b_{q}(n)$ we must compute the quantity $B_{n, k}$. We have

$$
B_{n, k}=\frac{n !}{k !} \sum_{s_{q}(k, n)}\left(\begin{array}{c}
k \\
k_{1}, \cdots, k_{n-k+1}
\end{array}\right) \prod_{r \in s_{q}} v_{r}^{k_{r}}
$$

The computation of the product $\prod_{r \in s_{q}} v_{r}^{k_{r}}$ gives

$$
\prod_{r \in s_{q}} v_{r}^{k_{r}}=(-2)^{k_{1}}(-1)^{k_{q}} 2^{k_{q+1}}(-1)^{k_{q+2}}=(-1)^{k_{1}+k_{q}+k_{q+2}} 2^{k_{1}+k_{q+1}}
$$

and then

$$
\prod_{r \in s_{q}} v_{r}^{k_{r}}=(-1)^{k-k_{2}-k_{q+1}} 2^{k_{1}+k_{q+1}}
$$

Furthermore

$$
B_{n, k}=\frac{n !}{k !} \sum_{s_{q}(k, n)}(-1)^{k-k_{2}-k_{q+1}} 2^{k_{1}+k_{q+1}}\left(\begin{array}{c}
k \\
k_{i}
\end{array}\right)_{i \in s_{q}} .
$$

Finally

$$
1 / A_{q}(t)=1+\sum_{n \geq 1} \sum_{k=1}^{n} \sum_{s_{q}(k, n)}(-1)^{k_{2}+k_{q+1}} 2^{k_{1}+k_{q+1}}\left(\begin{array}{c}
k \\
k_{i}
\end{array}\right)_{i \in s_{q}} t^{n},
$$

and the result follows.

2.2. Further identities of $b_{q}(n)$. We consider the functions $f(t)=\frac{1}{1-t}$ and $g(t)=t^{q}$, so we have

$$
\left(1-t^{q}\right)^{-1}=f \circ g(t) .
$$

The $n$-derivatives of $f$ and $g$ are respectively

$$
D^{n} f(t)=n !(1-t)^{-n-1} \text { and } D^{n} g(t)=(q)_{n} t^{q-n},
$$

where $(q)_{n}$ is the falling number given by

$$
(q)_{n}=\left\{\begin{array}{cc}
q(q-1) \cdots(q-n+1) & \text { if } n \leq q \\
0 & \text { if } n>q .
\end{array}\right.
$$

According to Faà di Bruno formula we have

$$
D^{n}\left(\left(1-t^{q}\right)^{-1}\right)=\sum_{k=0}^{n} k !\left(1-t^{q}\right)^{-k-1} B_{n, k}\left((q)_{1} t^{q-1},(q)_{2} t^{q-2}, \cdots\right) .
$$


But the $n$-th derivative of $f^{2}(t)$ is

$$
D^{n}(1-t)^{-2}=(n+1) !(1-t)^{-2-n} .
$$

We use the well-known Leibniz formula

$$
D^{n} f g(t)=\sum_{k=0}^{n}\left(\begin{array}{l}
n \\
k
\end{array}\right) D^{k} f(t) D^{n-k} g(t)
$$

to compute the $n$-th derivative of the product $f_{q}=f g$ :

$$
\begin{aligned}
D^{n} f_{q}(t) & =\sum_{u=0}^{n} \sum_{k=0}^{u}\left(\begin{array}{l}
n \\
u
\end{array}\right) k ! B_{u, k}\left((q)_{1} t^{q-1},(q)_{2} t^{q-2}, \cdots\right)(n-u+1) ! \\
& \times\left(1-t^{q}\right)^{-k-1}(1-t)^{-2-n+u} .
\end{aligned}
$$

But we have

$$
B_{u, k}\left((q)_{1} t^{q-1},(q)_{2} t^{q-2}, \cdots\right)=t^{q k-u} B_{u, k}\left((q)_{1},(q)_{2}, \cdots\right)
$$

Thus

$$
\begin{aligned}
D^{n} f_{q}(t) & =n ! \sum_{u=0}^{n} \sum_{k=0}^{u} \sum_{s_{u}(k)}(n-u+1)\left(\begin{array}{c}
k \\
k_{1} \cdots k_{u}
\end{array}\right) \prod_{r=1}^{u}\left(\frac{(q)_{r}}{r !}\right)^{k_{r}} \\
& \times\left(1-t^{q}\right)^{-k-1}(1-t)^{-2-n+u} t^{q k-u} .
\end{aligned}
$$

The following result follows from the fact that $\left.D^{n} f_{q}(t)\right|_{t=0}=b_{q}(n) n !$.

Theorem 2.3.

$$
b_{q}(n)=\sum_{u=0}^{n} \sum_{\substack{s_{u}(k) \\
q k=u}}(n-u+1)\left(\begin{array}{c}
k \\
k_{1} \cdots k_{q}
\end{array}\right) \prod_{r=1}^{u}\left(\frac{(q)_{r}}{r !}\right)^{k_{r}} .
$$

The two different forms (15) and (16) of $b_{q}(n)$ permit to conclude that

$$
\begin{gathered}
\sum_{u=0}^{n} \sum_{\substack{s_{u}(k) \\
q k=u}}(n-u+1)\left(\begin{array}{c}
k \\
k_{1} \cdots k_{q}
\end{array}\right) \prod_{r=1}^{u}\left(\frac{(q)_{r}}{r !}\right)^{k_{r}}= \\
\sum_{k=1}^{n} \sum_{s_{q}(k, n)}(-1)^{k_{2}+k_{q+1}} 2^{k_{1}+k_{q+1}} \cdot\left(\begin{array}{c}
k \\
k_{i}
\end{array}\right)_{i \in s_{q}} .
\end{gathered}
$$

\section{Vasyunin cotangent sums and Bell polynomials}

In this section we revisit the identity (1) to give an arithmetical proof different of that given in [21] which uses Fourier analysis and exponential sum. Let $\ell_{n}$ be the sequence defined by means of the generating function

$$
\frac{2-q+q t-q t^{q-1}+(q-2) t^{q}}{(1-t)^{2}\left(1-t^{q}\right)}=\sum_{n \geq 0} \ell_{n} t^{n},|t|<1 .
$$


First we give some recursive formulas satisfied by the sequence $\ell_{n}$. Letting

$$
2-q+q t-q t^{q-1}+(q-2) t^{q}=\sum_{n \geq 0} c_{n} t^{n}
$$

where $c_{n}$ is the sequence defined by $c_{0}=2-q, c_{1}=q, c_{q-1}=-q, c_{q}=q-2$ and the others are zeros. According to the identity

$$
\left(\sum_{n \geq 0} c_{n} t^{n}\right)\left(\sum_{n \geq 0} b_{q}(n) t^{n}\right)=\sum_{n \geq 0} \ell_{n} t^{n},
$$

we conclude that

$$
\ell_{n}=\sum_{k=0}^{n} c_{k} b_{q}(n-k)
$$

and for $n \geq q$ :

$$
\ell_{n}=(2-q) b_{q}(n)+q b_{q}(n-1)-q b_{q}(n-q+1)+(q-2) b_{q}(n-q) .
$$

Since we have

$$
b_{q}(n)=\sum_{k=1}^{n} \frac{k !}{n !}(-1)^{k} B_{n, k}
$$

Then

$$
\begin{aligned}
\ell_{n} & =(2-q) \sum_{k=1}^{n} \frac{(-1)^{k} k !}{n !} B_{n, k}+q \sum_{k=1}^{n-1} \frac{(-1)^{k} k !}{(n-1) !} B_{n-1, k} \\
& -q \sum_{k=1}^{n-q+1} \frac{(-1)^{k} k !}{(n-q+1) !} B_{n-q+1, k}+(q-2) \sum_{k=1}^{n-q} \frac{(-1)^{k} k !}{(n-q) !} B_{n-q, k} .
\end{aligned}
$$

Otherwise the identity

$$
\left(\sum_{n \geq 0} v_{n} t^{n}\right)\left(\sum_{n \geq 0} \ell_{n} t^{n}\right)=\sum_{n \geq 0} c_{n} t^{n}
$$

implies that

$$
c_{n}=\sum_{k=0}^{n} v_{k} \ell_{n-k}
$$

and for $n \geq q+2$ we have

$$
\ell_{n}=2 \ell_{n-1}-\ell_{n-2}+\ell_{n-q}-2 \ell_{n-q-1}+\ell_{n-q-2} .
$$

From the integral representation (2), it is easy to check that the Dirichlet series of $\pi V(1 / q)$ is

$$
\pi V(1 / q)=\pi \sum_{n \geq 0} \frac{\ell_{n}}{n+1}
$$


In what follows we propose a method to find the explicit formula of $\ell_{n}$. According to the identity

$$
\left(1-t^{q}\right)\left(\sum_{n \geq 0} \ell_{n} t^{n}\right)=\left(\sum_{n \geq 0} c_{n} t^{n}\right)\left(\sum_{n \geq 0}(n+1) t^{n}\right)
$$

we have

$$
\ell_{n}=\sum_{k=0}^{n}(n-k+1) c_{k}, n \leq q-1
$$

and

$$
\ell_{n}-\ell_{n-q}=\sum_{k=0}^{n}(n-k+1) c_{k}, n \geq q .
$$

Thus

$$
\ell_{n+q}-\ell_{n}=\sum_{k=0}^{n+q}(n+q-k+1) c_{k},
$$

but we have $\sum_{k=0}^{n+q}(n+q-k+1) c_{k}=0$. Then $\ell_{n+q}=\ell_{n}$ and $\ell_{n}$ is periodic of period $q$. To get all the values you just have to calculate $\ell_{r}$ for $0 \leq r<q-1$. In this case we have $\ell_{r}=2(r+1)-q$ for $r \leq q-2$ and $\ell_{q-1}=0$. Let $r$ be the remainder obtained when $\mathrm{n}$ is divided into $q$, then $n=k q+r$ and $\ell_{n}=\ell_{r}$. Thereafter

$$
\pi V(1 / q)=\sum_{k \geq 0} \sum_{r=1}^{q-1} \frac{2 r-q}{k q+r}
$$

Since we have $r=n-q\left\lfloor\frac{n}{q}\right\rfloor$ and $r$ is different of $q$ in the last sum, we deduce that

$$
\pi V(1 / q)=-\sum_{\substack{n \geq 1 \\ q \nmid n}} \frac{2\left(-n+q\left\lfloor\frac{n}{q}\right\rfloor\right)+q}{n}
$$

Finally we have

$$
c_{0}(1 / q)=-V(1 / q)=\frac{1}{\pi} \sum_{\substack{n \geq 1 \\ q \nmid n}}\left[\frac{q}{n}\left(1+2\left\lfloor\frac{n}{q}\right\rfloor\right)-2\right] .
$$

Acknowledgement. The authors would like to thank the referees for the valuable suggestions and comments.

\section{References}

[1] T. Apostol, Introduction to Analytic Number Theory, Springer-Verlag, New York, 1976.

[2] L. Báez Duarte, M. Balazard, M. Landreau and E. Saias, Etude de lutocorrélation multiplicative de la fonction partie fractionnaire, Ramanujan J., 9 (2005), 215-240. 
[3] A. Bayad and M. Goubi, Reciprocity formulae for generalized DedekindVasyunin-cotangent sums, Math. Methods Appl. Sci., 42(4) (2019), 1082-1098.

[4] S. Belhadj and M. Goubi, On the Vasyunin Cotangent sums related to Riemann Hypothesis, WSEAS Transactions on Mathematics, 19 (2020), 676-682.

[5] L. Comtet, Advanced Combinatorics, Reidel, Boston, 1974.

[6] F. Caldarola, M. Maiolo and V. Solferino, A new approch of Z-transform through infinite computation, Commun. Nonlinear Sci. Numer. Simulat., 82 (2020), article id. 105019.

[7] R. de la Bretèche and G. Tenenbaum, Séries trigonométriques à coefficients arithmétiques, J. Anal. Math., 92 (2004), 1-79.

[8] F. Faà di Bruno, Sullo Sviluppo delle funzioni, Annali di Scienze Matematiche e fisiche di Tortolini, 6 (1855), 479-480.

[9] T. Fukaya, Hasse Zeta Functions of Non-commutative Rings, J. Algebra, 208(1) (1998), 304-342.

[10] M. Goubi, Series expansion of a cotangent sum related to the Estermann zeta function, Kragujevac J. Mathematics, 45(3) (2021), 343-352.

[11] M. Goubi, On composition of generating functions, Casp. J. Math. Sci., 9(2) (2020), 256-265.

[12] M. Goubi, Explicit formula of a new class of q-Hermite-based Apostol-type polynomials and generalization, Notes on Number Theory and Discrete Mathematics, 26(4) (2020), 93-102.

[13] M. Goubi, A. Bayad and M. O. Hernane, Explicit and asymptotic formulae for Vasyunin-cotangent sums, Publ. Inst. Math. (Beograd) (N.S.), 102(116) (2017), 155-174.

[14] F. J. Grunewald, D. Segal and G. C. Smith, Subgroups of finite index in nilpotent groups, Invent. Math., 93(1) (1988), 185-223.

[15] M. Ishibashi, The value of the Estermann zeta function at $s=0$, Acta Arith., 73(4) (1995), 357-361.

[16] E. I. Jury, Theory and Application of Z-Transform Method, John Wiley \& Sons, New York, 1964.

[17] H. Maier and M. Th. Rassias, Generalizations of a cotangent sum associated to the Estermann zeta function, Commun. Contemp. Math., 18(1) (2016), 1550078 (89 pp).

[18] C. Papachristodoulos and M. Papadimitrakis, On universality and convergence of the Fourier series of functions in the disc algebra, J. Anal. Math., 137(1) (2019), 57-71. 
[19] J. Ragazzini and L. Zadeh, The analysis of sampled-data systems, Transactions of the American Institute of Electrical Engineers, Part II: Applications and Industry, 71(5) (1952), 225-234.

[20] M. Th. Rassias, Analytic Investigation of Cotangent Sums to the Riemann Zeta Function, Zurich, 2014.

[21] M. Th. Rassias, A cotangent sum related to zeros of the Estermann zeta function, Appl. Math. Comput., 240 (2014), 161-167.

[22] S. Roman, The Formula of Faà di Bruno, Amer. Math. Monthly, 87(10) (1980), 805-809.

[23] L. Solomon, Zeta functions and integral representation theory, Advances in Math., 26(3) (1977), 306-326.

[24] V. I. Vasyunin, On a biorthogonal system associated with the Riemann hypothesis, Algebra i Analiz, 7(3) (1995), 118-135.

[25] C. Voll, Functional equations for zeta functions of groups and rings, Ann. of Math., 172(2) (2010), 1181-1218.

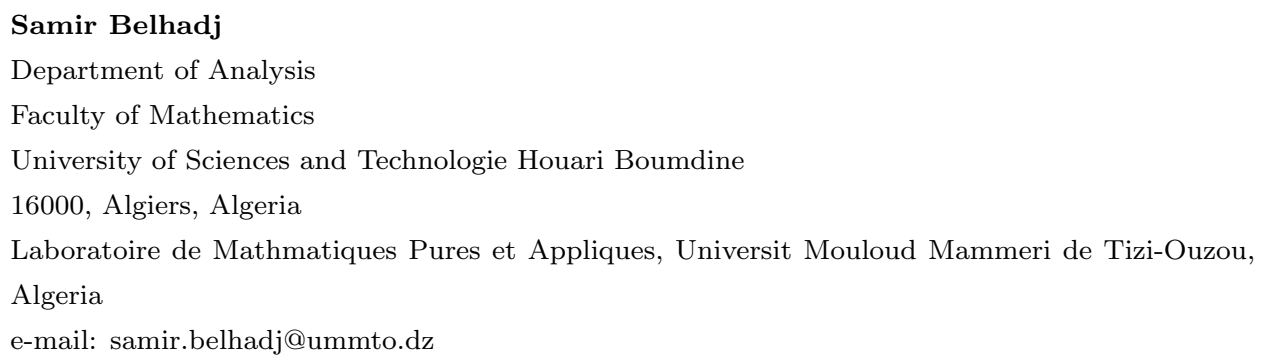

